\title{
Thin Basement Membrane Disease Associated With Nephrotic Range Proteinuria, Hypertension and Chronic Kidney Disease
}

\author{
Omkar U Vaidya ${ }^{\mathrm{a}}$, Essam F Elsayed ${ }^{\mathrm{a}, \mathrm{b}}$
}

\begin{abstract}
Thin basement membrane disease (TBMD) is typically associated with hematuria but proteinuria is an uncommon presentation. When proteinuria is present, it is mostly in a non-nephrotic range, moreover, when proteinuria is in nephrotic range, generally it is associated with presence of glomerulopathies, Focal Segmental Nephrosclerosis, IgA Nephropathy or minimal change and is rarely present in an isolated TBMD. We present a case of 53-year-old male with history of hypertension for $15 \mathrm{yrs}$, coronary artery disease, rheumatoid arthritis and stage III chronic kidney disease who was referred to us for evaluation of long-standing chronic microscopic hematuria and new onset nephrotic range proteinuria. Given the fact that the proteinuria was new and in nephrotic range a biopsy was performed and was consistent with TBMD without other glomerlopathies or any other abnormalities seen. Our patient had CKD stage III serum (creatinine1.2 - $1.6 \mathrm{mg} / \mathrm{dL}$ ), which was a stable finding for past 12 years. Microscopic hematuria was persistent for 24 years with new onset (6 month) of proteinuria. This case of TBMD is unique in terms of new onset nephrotic range proteinuria after long standing hematuria for 24 years.
\end{abstract}

Keywords: Hematuria; Proteinuria; Chronic kidney disease

\section{Introduction}

TBMD sometimes referred as benign familial hematuria is characterized by thinning of basement membrane $<250 \mathrm{~nm}$ without involvement of other renal compartments, absence

Manuscript accepted for publication May 27, 2013

\footnotetext{
${ }^{a}$ Division of Nephrology, Veterans Affairs North Texas and UT Southwestern Medical School, USA

${ }^{\mathrm{b}}$ Corresponding author: Essam F Elsayed, Dallas VA Medical Center, 4500 S Lancaster Road, Dallas, TX 75216, USA.

Email: Essam.elsayed@va.gov
}

doi: http://dx.doi.org/10.4021/wjnu97w of family history of deafness and/or renal disease [1-3]. TBMD is seen in $5-10 \%$ of general population but a wide range of prevalence was reported in literature. Proteinuria has been reported in about $60 \%$ of patients with TBMD but is usually mild and most likely $<500 \mathrm{mg} / \mathrm{dL}$ per day but rarely it can present with nephrotic range proteinuria, worsening kidney function and hypertension.

\section{Case Report}

A 53-year-old male with a history of hypertension for $15 \mathrm{yrs}$, coronary artery disease and rheumatoid arthritis (diagnosed 2 yrs ago) was referred to us for evaluation of chronic microscopic hematuria and new onset nephrotic-range proteinuria. He was first noted to have microscopic hematuria when he was 24 years old (29 years ago); further evaluations at that time, including cystoscopy was unremarkable. As per records urinanalysis (UA) done 12 years ago also showed hematuria and $2+$ proteinuria (10 years prior to diagnosis of RA). Subsequent UA showed persistent hematuria and mild proteinuria. Serum creatinine was within normal limit till after his myocardial infarction (was exposed to Intravenous iodine contrast at that time). He developed kidney injury with a baseline serum creatinine between $1.2-1.6 \mathrm{mg} / \mathrm{dL}$. Patient denies having gross hematuria, flank pain, urinary tract infections or other urinary symptoms and there is no skin rash or pulmonary symptoms. Review of systems was negative other than chronic arthralgia. Physical exam with stable vital signs showed that the patient was afebrile with temperature $36.9^{\circ} \mathrm{C}$, blood pressure $143 / 87$, heart rate 71 , oxygen saturation of $97 \%$, weight $221 \mathrm{lb}(100.5 \mathrm{~kg})$ and BMI of 30, otherwise no significant clinical findings except mild swelling of MCP joints of both hands.

Past medical history as above, and significant for seropositive non-erosive RA well controlled and responded well to Abatacept, which he started 9 months ago and last infusion was about 3 months ago and currently he is on hydroxychloroquine, and prednisone. No family history of kidney disease or deafness. He is a current tobacco user and works in an office environment.

Laboratory studies were significant for Serum creatinine 


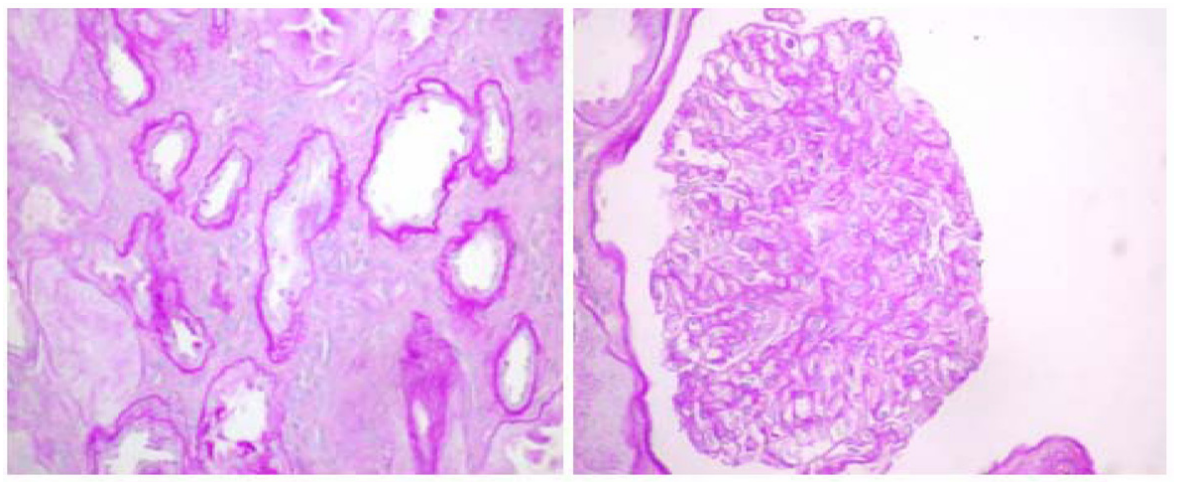

Figure 1. Light Microscopy with periodic acid-Schiff (PAS) stain shows non-specific abnormalities $(600 \times s)$.

of $1.37 \mathrm{mg} / \mathrm{dL}$; Albumin $1.9 \mathrm{~g} / \mathrm{dL}$ (was noted to be normal one year ago); 24 hour urine with $5.6 \mathrm{~g} /$ day of proteins. Antinuclear Antibody (ANA) was negative as well as hepatitis profile, including HBV, HCV as well as negative HIV. Renal ultrasound done 6 months ago was unremarkable, with normal sized kidneys, no hydronephrosis or stones. Giving the wide differential diagnosis and possibility of IgA nephropathy or less likely a combination of a nephrotic and a nephritic glomerulopathy, we decided to perform a kidney biopsy.

\section{Kidney biopsy results}

Light microscopy depicted 9 glomeruli of which five showed minimal lesions and three exhibited some mild focal global sclerosis and one was obsolete (these are located in the proximity of arteriolosclerotic scars). Arterioles revealed intimal fibroelastic thickening. Interstitial fibrosis or tubular atrophy did not exceed $15 \%$ of the parenchyma (Fig. 1).

On immunofluorescence (8 glomeruli); Albumin, IgG, IgA, IgM, C3, C4, C1q, Kappa, Lambda and fibrin staining were all negative.

On Electron Microscopy (EM) there was extensive thinning of glomerular basement membrane. Mean thickness was 243 nanometers (measured by the orthogonal intersection method-normal $300 \pm 50$ ) (Fig. 2).

\section{Discussion}

According to WHO guidelines TBMD is diagnosed if there is diffuse Glomerular Basement Membrane (GBM) thinning less than $250 \mathrm{~nm}$ [3] in adults and $180 \mathrm{~nm}$ if in children. GBM thickness progressively increases in children aged 8 11 years and then plateaus after that [3].

TBMD can be an autosomal dominant disorder and in $40 \%$ of families and segregates with COL 4 alfa 3 , COL 4 alfa 4 loci. Various types of genetical mutations like, missense mutation, nonsense mutation, splice mutations, deletions and insertions have all been described in Alports and TBDM [4]. Alports syndrome and TBMD can be considered as genetic disease of GBM involving type IV collagen [5].

Alport syndrome (AS) is characterized by renal, cochlear, and ocular involvement. Renal disease progresses from microscopic hematuria to proteinuria, progressive renal insufficiency, and end-stage renal disease (ESRD) in all males with X-linked (XL) AS, and in all males and females
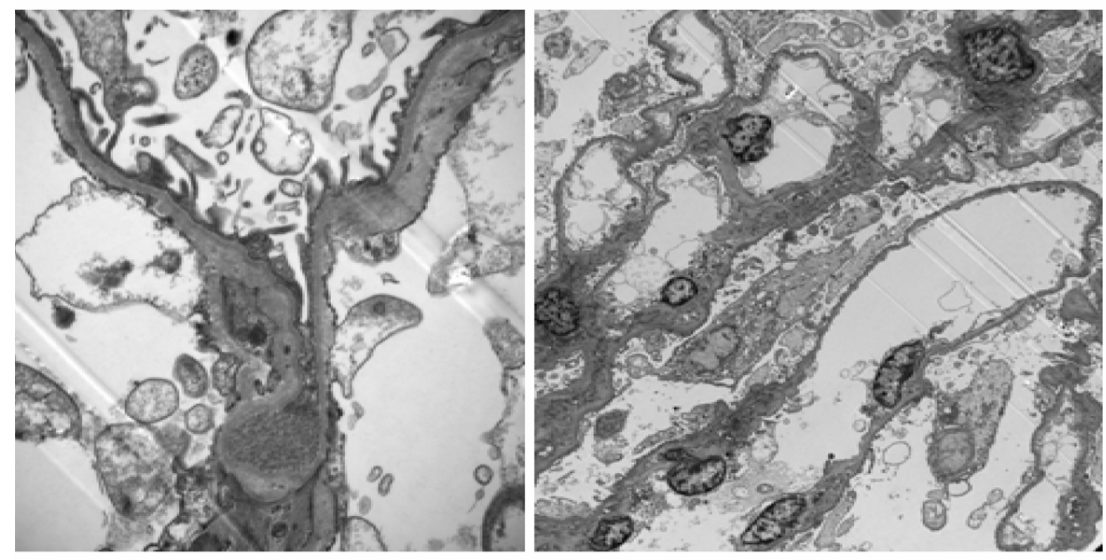

Figure 2. Electron Microscopy: shows diffuse thinning of glomerular basement membrane $(15,000 \times)$ 
with autosomal recessive (AR) [5]. TBMD is characterized by persistent microscopic hematuria often first observed in childhood; progressive renal involvement and extrarenal abnormalities are rare [5]. TBMD is a disease of genetic heterogeneity; it is not a benign renal condition in a substantial number of patients, particularly those in late middle age and can be diffuse or segmental $[4,6]$.

Dishe et al. described series of 14 patients, 3 patients with TBM, two proteinuric patients had nephrotic range and one had $2.5 \mathrm{~g} / \mathrm{dL}$ [6]. Nogueira $\mathrm{M}$ et al described case series of TBMD with heavy proteinuria. It was noted only in three patients that had TBM and the rest had other glomerulopathies like FSGS. Of the three patients, two were treated with steroids with good response. One patient who was not treated had persistent proteinuria and thought to be associated with Minimal change disease [7]. Abe et al. presented a series of 8 cases where all had stable renal function and benign prognosis [8]. Ogawa et al. described a case where an $80 \mathrm{yr}$ old female with scleroderma, mild hematuria and $0.9 \mathrm{~g}$ proteinuria with progression to $12.4 \mathrm{~g} /$ day was diagnosed with minimal change disease on second renal biopsy [9]. Van Paassen et al conducted a prospective study in the southern part of Netherlands that showed that the vast majority of TBMD had chronic microscopic hematuria, frequently associated with hypertension in late middle age; about $15 \%$ of TBMD had in addition substantial proteinuria which is associated in the majority of cases with the lesions of FSGS. In $5 \%$ of TBMD a nephrotic syndrome is observed, occasionally associated with FSGS tip lesions.

Over all from the literature it has been established that TBMD is a benign prognosis disease. Proteinuria associated with it is not common and in most cases proteinuria is mild, when heavy proteinuria is present, it is associated with other glomerulopathies like FSGS, MCD, IgA. The findings of global sclerosis on kidney biopsy in patients with TBMD has been previously reported as these patients also develop premature glomerular sclerosis $[10,11]$, which might be in early stages of development in our patient and might contribute to recent increase in proteinuria.

Our patient has stage III CKD which was a stable finding for past 12 years. He had hematuria for 24 years as per our records and nephrotic range proteinuria for past 6 months only. However, cause of CKD unclear at this time but could be related to prior use of NSAIDS or medications used to treat RA, or contrast induced nephropathy or long standing hypertension, interestingly, both hypertension and CKD are reported to be associated with some cases of TBMD $[8,10$ 12]. As there was no evidence of glomerulonephritis, MCD on kidney biopsy we elected not to start the patient on immunosuppressants and started him on lisinopril and advised to avoid NSAIDS. At this time our plan is to treat him conservatively with ACE inhibitors and repeat labs including 24 hours urine in 3 months and at the time will reconsider another biopsy if no significant improvement in proteinuria as some reported cases of MCD associated with TBMD that required more than one biopsy for diagnosis [9].

In conclusion, this is an unusual case of nephrotic range proteinuria, hypertension and CKD after long standing microscopic hematuria for 24 years with kidney biopsy showing TBMD with some mild focal global sclerososis. It also demonstrates the importance of collaboration between nephrologists and urologists. As in this case, many times urologist are being consulted first in cases presenting with microscopic hematuria but they should make sure to get a nephrology input when these cases also have proteinuria or CKD or HTN as they might require kidney biopsy and management with close follow up.

\section{References}

1. Yoshikawa N, Cameron AH, White RH. The glomerular basal lamina in hereditary nephritis. J Pathol. 1981;135(3):199-209.

2. Foster K, Markowitz GS, D'Agati VD. Pathology of thin basement membrane nephropathy. Semin Nephrol. 2005;25(3):149-158.

3. Aarons I, Smith PS, Davies RA, Woodroffe AJ, Clarkson AR. Thin membrane nephropathy: a clinico-pathological study. Clin Nephrol. 1989;32(4):151-158.

4. Savige J, Rana K, Tonna S, Buzza M, Dagher H, Wang YY. Thin basement membrane nephropathy. Kidney Int. 2003;64(4):1169-1178.

5. Kashtan CE. Alport Syndrome and Thin Basement Membrane Nephropathy: Diseases Arising from Mutations in Type IV Collagen. Saudi J Kidney Dis Transpl. 2003;14(3):276-289.

6. Dische FE, Weston MJ, Parsons V. Abnormally thin glomerular basement membranes associated with hematuria, proteinuria or renal failure in adults. Am J Nephrol. 1985;5(2):103-109.

7. Nogueira M, Cartwright J, Jr., Horn K, Doe N, Shappell $\mathrm{S}$, Barrios R, Coroneos E, et al. Thin basement membrane disease with heavy proteinuria or nephrotic syndrome at presentation. Am J Kidney Dis. 2000;35(4):E15.

8. Abe S, Amagasaki Y, Iyori S, Konishi K, Kato E, Sakaguchi H, Shimoyama K. Thin basement membrane syndrome in adults. J Clin Pathol. 1987;40(3):318-322.

9. Ogawa R, Miyoshi K, Nagao T, Jotoku M, Irita J, Okura T, Higaki J. [Ultrastructure of glomerular podocyts in the incipient phase of minimal change nephrotic syndrome with thin basement membrane disease]. Nihon Jinzo Gakkai Shi. 2012;54(8):1192-1196.

10. Frasca GM, Onetti-Muda A, Mari F, Longo I, Scala E, Pescucci C, Roccatello D, et al. Thin glomerular basement membrane disease: clinical significance of a morphological diagnosis--a collaborative study of the Italian Renal Immunopathology Group. Nephrol Dial Trans- 
plant. 2005;20(3):545-551.

11. Nieuwhof CM, de Heer F, de Leeuw P, van Breda Vriesman PJ. Thin GBM nephropathy: premature glomerular obsolescence is associated with hypertension and late onset renal failure. Kidney Int. 1997;51(5):1596-1601.
12. van Paassen P, van Breda Vriesman PJ, van Rie H, Tervaert JW. Signs and symptoms of thin basement membrane nephropathy: a prospective regional study on primary glomerular disease-The Limburg Renal Registry. Kidney Int. 2004;66(3):909-913. 